\title{
ERA-Erfahrungen in Thüringen - Befunde aus Pilotbetrieben
}

Das momentan bundesweit in der Metall- und Elektroindustrie umzusetzende Entgeltrahmenabkommen (ERA) wird zu Recht häufig als Jahrhundertreform bezeichnet, denn es hebt die lange Zeit herrschende Trennung von Arbeitern und Angestellten auf, sowohl was die Arbeitsbewertung als auch die Entgeltgrundlagen und die Anwendung von Leistungsentgelten angeht. Das Tarifgebiet Thüringen gehört in zeitlicher Hinsicht zu den Vorreitern der Umsetzung. Nicht nur aus diesem Grund eignete es sich für eine sozialwissenschaftliche Untersuchung der ERA-Einführung - am Beispiel Thüringen fällt auch Licht auf die Besonderheiten ostdeutscher Regionen sowie auf die Wirkung thüringenspezifischer Sonderregelungen.

\section{1 \\ ERA-Umsetzung: Enorme Herausforderung}

Ein Jahrhundertwerk wie das Entgeltrahmenabkommen (ERA) (Meine et al. 2006) stellt für die Tarifparteien und die Verhandlungspartner auf Betriebsebene - Geschäftsleitungen und Betriebsräte -, aber auch für die Belegschaften selbst eine große Herausforderung dar. Werden doch mit der ERA-Umsetzung die überlieferten Arbeitsbewertungen, Entgeltstrukturen und Relationen der Belegschaftsgruppen zueinander in allen Betrieben neu austariert und verhandelt. Die langjährig stabilen Unterschiede in der Bewertung der Arbeit von Arbeitern und Angestellten stehen zur Disposition. ERA setzt so an den Grundfesten der bisherigen Gratifikationsstrukturen und Reproduktionschancen von Arbeitnehmern bzw. der Kostenstrukturen der Verbandsmitglieder an, indem in jedem Betrieb die Arbeitsanforderungen jedes einzelnen Arbeitnehmers durch den Arbeitgeber neu bewertet und mit dem Betriebsrat abgestimmt werden. Wie Betriebe in Thüringen mit dieser Herausforderung umgegangen sind und wie sie ERA umgesetzt haben, behandelt der vorliegende Beitrag. Er stellt Kernbefunde der sozialwissenschaftlichen Begleitforschung des ISF München zur ERA-Einführung im Tarifgebiet Thüringen vor. ${ }^{1}$

Angesichts der weitreichenden Konsequenzen der ERA-Umsetzung - insbesondere auch für die Einkommen der Arbeitnehmer - kann als Ausgangsthese festgehalten werden: Es sind sowohl Konflikte zwischen Arbeitgebern und Arbeitnehmer- vertretung als auch Streitigkeiten zwischen unterschiedlichen Beschäftigtengruppen zu erwarten. Die diesem Beitrag zugrunde liegende Untersuchung ergab den Befundso viel sei vorweggenommen -, dass der Einführungsprozess in den untersuchten Betrieben zwar nicht konfliktfrei, aber doch überraschend einvernehmlich über die Bühne gegangen ist. Erklärungsansätze für dieses überraschende Ergebnis werden hier diskutiert.

Die empirischen Befunde der Untersuchung bieten auch Anhaltspunkte für Antworten auf weitere Fragen: Inwieweit ist in Thüringen eine Sondersituation gegeben einerseits als ostdeutsche Tarifregion, andererseits wegen der spezifischen Regelung der „Tariflichen Entsprechung“? Haben sich die Hoffnungen von Arbeitgeberverband und Gewerkschaft auf eine Revitalisierung des Flächentarifvertrags und eine Stärkung der Tarifparteien erfüllt? Wie sind die Chancen der Gewerkschaft zu beurteilen, mit ERA neue Mitglieder zu gewinnen?

Im Folgenden wird zunächst auf die Kennzeichen und Besonderheiten des ERATarifwerks für Thüringen eingegangen (Abschnitt 2). Es folgt eine Kurzfassung der empirischen Befunde zum Einführungsprozess in drei Pilotbetrieben sowie zur Betroffenheit unterschiedlicher Beschäftigtengruppen (Abschnitt 3). Abschnitt 4 gibt Gesamtbewertungen von ERA seitens der Gesprächspartner wieder und skizziert die Folgen für den Rückhalt der Betriebsräte und Gewerkschaften in den Belegschaften. Den Abschluss bildet ein Resümee (Abschnitt 5), das die empirischen Erkenntnisse vor dem Hintergrund von Trendaussagen und Thesen der Forschung zu industriellen Beziehungen bilanziert.

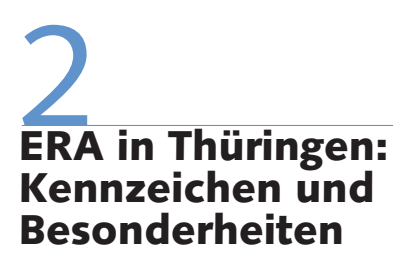

Im Vergleich zu anderen Tarifbezirken und -gebieten wurde ERA in Thüringen relativ frühzeitig abgeschlossen, nämlich im Januar 2004 - mit einer Umsetzungsfrist zwischen dem 1. Januar 2006 und dem 31. Dezember 2007. Auf freiwilliger Basis und mit Zustimmung der Tarifvertragsparteien, der IG Metall und des Arbeitgeberverbands VMET waren Abweichungen möglich, d.h. frühere Einführung oder Verlängerung der Umsetzungsfrist bis zum 31. Dezember 2008.

Die Tarifvertragswerke zu ERA in Thüringen sehen eine Eingruppierung der Beschäftigten entsprechend ihren Tätigkeitsanforderungen in zwölf Entgeltgruppen (E 1 bis E 12) vor. Die Eingruppierung rich-

\footnotetext{
1 Das zugrunde liegende Projekt "Sozialwissenschaftliche Begleitforschung zur ERA-Einführung im Bereich der Metall- und Elektroindustrie Thüringens" wurde im Auftrag der Otto Brenner Stiftung von April 2005 bis Mai 2008 durch das Institut für Sozialwissenschaftliche Forschung e. V. - ISF München durchgeführt (vgl. den ausführlichen Bericht Schmierl 2008).
} 
tet sich nach den summarischen Anforderungsmerkmalen des Entgeltrahmenabkommens und erfolgt nach derjenigen Tätigkeit, die das Niveau der Gesamttätigkeit prägt, sodass eine ganzheitliche Betrachtung der Anforderungen vorzunehmen ist - es ist also nicht mehr der zeitliche Umfang der Tätigkeit maßgebend. Zu jeder Entgeltgruppe ist eine Zusatzstufe ( $\mathrm{Z} 1$ bis $Z$ 12) vorgegeben, die eine höhere Entlohnung für die dauerhafte Übernahme dispositiver, Leitungs- oder Führungsaufgaben vorsieht.

Eine Sonderregelung für Thüringen stellt das tariflich vereinbarte Instrument der "Tariflichen Entsprechung“ dar. Es räumt den betrieblichen Arbeitgebern die Möglichkeit ein, zur Ermittlung der neuen Eingruppierungen eine Analogietabelle mit Nennung der alten Lohn- bzw. Gehaltsgruppe und der neuen Entgeltgruppe (als pauschales Äquivalent) anzuwenden. Die Alternative zur „Tariflichen Entsprechung" besteht in einer Neubewertung aller Arbeitsplätze/Arbeitskräfte des Betriebs.

Mit der "Tariflichen Entsprechung“ haben die thüringischen Tarifvertragsparteien einen entscheidenden Vorgriff auf die veränderten Eingruppierungsrelationen getätigt: Sie haben sich auf eine neue Relation der Entgeltgruppen zueinander in Gestalt einer Entgelttabelle geeinigt. Der alte Tarif sah im Arbeiterbereich neun Lohngruppen und im Angestelltenbereich jeweils sechs K- und T-Gruppen (kaufmännische und technische Angestellte) sowie vier Meistergruppen vor. Die Tarifliche Entsprechung führt diese Lohn- und Gehaltsgruppen in die für alle Beschäftigtengruppen einheitlichen Entgeltgruppen von ERA über. Zwei neue Entgeltgruppen ohne Entsprechung im alten Tarif, E 9 und E 11, bringen eine Spreizung der Eingruppierung im oberen Bereich mit sich und ermöglichen eine stärkere Differenzierung bei den höheren, akademisch geprägten bzw. leitenden Positionen.

Im Hinblick auf die neuen Grundsätze und Methoden der Entgeltgestaltung erlaubt der ERA-Tarifvertrag drei unterschiedliche Modelle: Zeitentgelt mit Beurteilung, Leistungsentgelt mit Kennzahlenvergleich und Leistungsentgelt mit Zielvereinbarung. Bei allen Entgeltgrundsätzen ist eine individuelle oder gruppenbezogene Ermittlung zulässig.

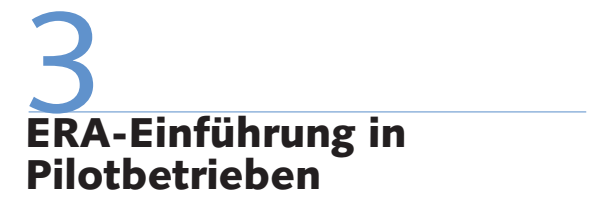

Die Begleitforschung wurde vom Autor in Abstimmung mit der IG Metall Bezirksleitung und dem Thüringer Arbeitgeberverband VMET zwischen 2005 und 2008 durchgeführt. Methodisch wurden in einer Bestandsaufnahme in zwei Wellen (Sommer 2006 und Sommer 2007) und im Rahmen leitfadengestützter qualitativer Experteninterviews mit Vertretern der Personalleitung und des Betriebsrats sowie in Interviews mit betroffenen Beschäftigten die Aushandlungsprozesse zwischen Personalleitung und Betriebsräten sowie die betrieblichen Umsetzungslösungen in den Vorreiterbetrieben des Tarifgebiets erhoben. Hierfür waren in Thüringen günstige Bedingungen gegeben, da es sich um ein überschaubares Tarifgebiet mit ca. 50 größeren, stabil mitbestimmten und tarifgebundenen Unternehmen handelt, die zusammen ca. 16.000 Arbeitnehmer beschäftigen (von insgesamt 79.000 Beschäftigten im Tarifgebiet) (vgl. IWT 2007). Tarif- und sozialpolitisch werden diese Unternehmen vom Verband der Metall- und Elektroindustrie in Thüringen e. V. (VMET) mit Sitz in Erfurt vertreten; er repräsentierte im Jahr 2006 19,3 \% der in dieser Branche Beschäftigten. Auf die Gesamtheit der Unternehmen bezogen, unterliegen jedoch nur $6 \%$ der Tarifbindung, nämlich 56 Unternehmen. Vorwiegend im Feld der Dienstleistungen ist zudem der 1993 gegründete Allgemeine Arbeitgeberverband Thüringen e. V. (AGVT) als tariffreier und branchenübergreifender Verband tätig; aus der Metallindustrie sind in diesem OT-Verband weitere 40 Unternehmen Mitglied, was etwa $5 \%$ der Metall-Unternehmen in Thüringen entspricht (Haipeter/Schilling 2006). Seitens der Gewerkschaften ist die IG Metall Bezirksleitung in Frankfurt/Main für das Tarifgebiet der Metall- und Elektroindustrie Thüringens als Tarifpartner zuständig.

Für die ERA-Begleitforschung in Thüringen wurden drei Unternehmen aus der Gesamtheit der tarifgebundenen Arbeitgeber des Tarifbezirks ausgewählt. Dafür wurden exemplarische Typen von Pilotbetrieben identifiziert, die durch jeweils spezifische Bedingungen (Teilbranche und Produktspektrum, Betriebsgröße, Belegschaftsstruktur) charakterisiert sind und als repräsentativ für Betriebe mit ähnlichen betriebsstrukturellen Bedingungen betrachtet werden können:

- ein Großunternehmen aus der Optikindustrie mit einer gemischten Belegschaftsstruktur aus Gewerblichen und Angestellten;

- ein stärker produktionsgeprägtes Unternehmen aus der Automobilindustrie mit einem hohen Anteil von Produktionsarbeitern;

- ein Klein- bzw. Mittelbetrieb (KMU) aus der Optikindustrie mit sehr hohem Angestelltenanteil.

Das Forschungsdesign zielte also nicht auf eine quantitative, statistisch repräsentative Erhebung, sondern auf eine in die Tiefe gehende qualitative Analyse der typischen Problembedingungen sowie der spezifischen Wechselwirkungen und Folgen von ERA im Betrieb.

\subsection{ANWENDUNG DER TARIFLICHEN ENTSPRECHUNG}

Die drei Untersuchungsbetriebe haben zum frühestmöglichen Zeitpunkt ERA eingeführt. Sie stellen sowohl hinsichtlich des Umstellungszeitpunkts als auch hinsichtlich der internen Informationspolitik „Leuchtturmbetriebe“ dar. Alle Betriebsparteien gingen bei der Entscheidung über die ERAUmsetzung nach der "Tariflichen Entsprechung" davon aus, dass die vorgängigen Arbeitsbewertungen und Entgeltstrukturen der betrieblichen Realität angemessen sind und zutreffen $-z$. B., weil vor nicht allzu langer Zeit eine Arbeitsbewertung der betrieblichen Arbeitsprozesse oder eine Aktualisierung der Funktionsbeschreibungen stattgefunden hatte.

Als erster Schritt der Koordinierung wurde in allen drei Fällen die Betriebsvereinbarung zur Anwendung der „Tariflichen Entsprechung" abgeschlossen. Nach der Einzelfallprüfung der neuen Entgeltrelationen zeigte sich, dass Differenzierungen notwendig waren. Dies führte zu einem jeweils betriebsspezifischen Umgang: Während bei dem Unternehmen aus der Automobilindustrie schnell erkennbar war, dass die "Tarifliche Entsprechung" für die gesamte Belegschaft angewendet werden konnte, stellte sich in den beiden Betrieben aus der Optikindustrie heraus, dass eine begrenzte Abkehr von der vollständigen Anwendung erforderlich war. Aufgrund 
struktureller Veränderungen seit der letzten Eingruppierungsrunde mussten hier Neueingruppierungen vorgenommen werden, nämlich für die Mitarbeiter aus Abteilungen, die technologischen Veränderungen unterworfen waren. In beiden Fällen konnten schließlich ca. $85 \%$ bis $90 \%$ der Belegschaft nach den Maßgaben der ,Tariflichen Entsprechung" übergeführt werden, während die restlichen ca. $10 \%$ bis $15 \%$ im Zuge der ERA-Einführung neu eingruppiert wurden.

\subsection{BESONDERE PROBLEME DER TARIFVERTRAGSANWENDUNG}

Bei der Anwendung des ERA-Tarifvertrags zeichneten sich in allen Untersuchungsbetrieben ähnliche Probleme ab:

(1) Die neuen Zusatzstufen (Z-Stufen) stehen Beschäftigten zu, die zusätzlich dauerhaft dispositive Aufgaben und/oder Aufgaben der Anleitung und Führung von Beschäftigten ausführen oder denen dauerhaft zusätzliche Tätigkeiten übertragen werden, die wesentlich über die Anforderungen der Entgeltgruppe hinausgehen. Nach Einschätzung der Gesprächspartner sind diese Bedingungen im ERA-Tarifvertrag nicht eindeutig geregelt; so stellten sich in der betrieblichen Anwendung u.a. folgende Fragen: Welche dispositiven Funktionen rechtfertigen eine Eingruppierung in eine der zwölf Z-Stufen? Wie ist bei einer zeitlich befristeten Übernahme dieser Funktionen zu verfahren? Angesichts solcher Unklarheiten tendierten die Untersuchungsbetriebe zu einem sehr zurückhaltenden Umgang mit den Zusatzstufen; in einer Firma wurden die Z-Stufen überhaupt nicht vergeben, weil man die Erfahrungen mit der ERA-Anwendung im Betrieb abwarten wollte.

(2) Berechnungsmodus und Ausschüttung bei Abweichungen zwischen den Bruttoentgelten: Sowohl bei Anwendung der ,Tariflichen Entsprechung" als auch bei Neueingruppierungen können sich die neuen Entgelte der Beschäftigten von den alten Entgelten unterscheiden:

Bei den Überschreitern überstiege das alte Gehalt das neue Entgelt, wenn die neuen Eingruppierungsregelungen vollständig in Kraft gesetzt würden - ihr neues Entgelt wäre daher niedriger als das alte. Die Besitzstandsregelung im Tarifvertrag verhindert eine solche Entgeltkürzung, er- möglicht allerdings eine Anrechnung auf künftige Tariferhöhungen. Das heißt: Die Überschreiter erhalten weiterhin ihre vorgängige Lohn- oder Gehaltssumme. Die rechnerische Differenz zwischen Alt und Neu wird als „Ausgleichszulage“ auf künftige Tariferhöhungen so lange angerechnet, bis sie abgeschmolzen ist. An Tariferhöhungen partizipieren sie damit für diesen Zeitraum nicht in Form einer Einkommenssteigerung, die dem vollen Volumen der Tariferhöhung entspricht.

Bei den Unterschreitern würde umgekehrt das alte Gehalt das neue Entgelt unterschreiten; ihr neues Entgelt wäre also höher als das alte. Um die Betriebe - aus der Interessenlage des Arbeitgeberverbands vor sprunghaft steigenden Entgeltsummen zu schützen und um - aus der Interessenlage der Gewerkschaft - zu verhindern, dass die Betriebe Höhergruppierungen vermeiden, sieht der Tarifvertrag eine schrittweise Anhebung der Entgelte von Unterschreitern vor: Spätestens nach 60 Monaten ist demzufolge die vollständige Anpassung an das Entgelt des Entgeltrahmenabkommens erreicht.

Die empirischen Untersuchungen ergaben, dass die Anpassung relativ schnell verlief und im Normalfall bereits im Sommer 2007, für den Großteil der restlichen Fälle mit der Tariferhöhung 2008 abgeschlossen war. Nur in wenigen Ausnahmeund Einzelfällen waren längere Korrekturphasen erforderlich.

(3) Eine zentrale Rolle spielten die Paritätischen Kommissionen. Die ERA-Tarifwerke schreiben die Einrichtung dieses Gremiums für Meinungsverschiedenheiten in zwei Fällen vor: bei Reklamationen im $\mathrm{Zu}$ sammenhang mit den neuen Grundsätzen der Entgeltgestaltung und Fragen der Leistungsermittlung sowie hinsichtlich der Arbeitsbewertung und Eingruppierung. Widersprüche können sowohl von den Betriebsräten als auch den Beschäftigten selbst eingelegt werden. Die Paritätischen Kommissionen setzen sich aus vier betriebsangehörigen Mitgliedern zusammen, die zur Hälfte vom Arbeitgeber und zur Hälfte vom Betriebsrat benannt werden. Die Untersuchungsbetriebe nutzten dieses Instrument zur Beilegung von Meinungsverschiedenheiten bereits in der Anfangsphase der ERA-Einführung sehr intensiv - speziell beim Thema der Über- bzw. Unterschreiter. Sie wurden also offenbar als sinnvolle Instanz zur Konfliktlösung angesehen.
(4) Die Verfahren der Eingruppierung bereiteten insbesondere aufgrund der Widerspruchsbereinigung in den Paritätischen Kommissionen keine größeren Probleme. Verzögerungen prägten hingegen die Umsetzung der ERA-Richtlinien zu den neuen Entgeltgrundsätzen und-methoden. In den beiden kleineren Untersuchungsfällen mit einer weitgehend homogenen Belegschaft wurde bereits vor der ERA-Umsetzung ein einheitlicher Entgeltgrundsatz (Lohn bzw. Gehalt mit Leistungszulage) angewandt daher war eine zeitnahe Anpassung der Entgeltgrundsätze nach der betrieblichen Entscheidung für eine Vereinheitlichung der Leistungszulage für alle Beschäftigten möglich. Dagegen ist in dem heterogeneren Großunternehmen mit vormals drei unterschiedlichen Entgeltgrundsätzen die vollständige Umsetzung des ERA-Tarifvertrags nicht für die komplette Belegschaft gelungen, sondern nur für etwa $80 \%$. Insbesondere für die Leistungslöhner im vormals gewerblichen Bereich deutete sich die Schwierigkeit an, deren bisher $\mathrm{z}$. T. höheres individuelles Gesamteinkommen, das durch die Leistungslohnkomponente zustande kam, in einen im gesamten Unternehmen einheitlichen Entgeltgrundsatz zu überführen.

(5) Ein gewisses Konfliktpotenzial für die Zukunft birgt der Umsetzungsbedarf im Hinblick auf die Errechnung und Ausschüttung des Strukturanpassungsfonds: Während die Ausschüttung des Gesamtvolumens der ERA-spezifischen Entgeltkosteneinsparung beim Automobilbetrieb bereits unmittelbar mit dem Einführungstermin im Jahr 2006 vorgenommen wurde, sind in den beiden anderen Untersuchungsbetrieben noch entsprechende Vorgehensweisen zu definieren.

\subsection{BETROFFENHEIT UNTERSCHIED- LICHER BESCHÄFTIGTENGRUPPEN}

Als begünstigte Beschäftigtengruppen sind an erster Stelle diejenigen Gruppen zu nennen, die im Rahmen der Anwendung der Tariflichen Entsprechung oder Neueingruppierung eine Höhergruppierung erfahren haben oder als Unterschreiter künftig mehr Einkommen zur Verfügung haben werden. Vorteile haben besonders die niedrigeren ehemaligen K-Gehaltsgruppen (Sekretärinnen und Teamassistentinnen sowie die niedrigeren kaufmännischen Angestellten), aber auch eine Reihe von gewerblichen Mitarbeitern aus Produktion und 
Montage in Unternehmen mit Unterschreitern. Zudem deutete sich eine Verbesserung der Einkommenssituation von neu eingestellten Hochschulabsolventen insofern an, als diese aufgrund der Ausdifferenzierung der höheren Entgeltgruppen nun eine bessere Eingangseingruppierung erhalten. Auch für Ingenieure sind durch die neuen, zwischengeschalteten Entgeltgruppen nun bessere Aufstiegschancen vorhanden.

Als benachteiligte Beschäftigtengruppen können sich die Überschreiter verstehen. Nicht deshalb, weil sie absolut weniger Entgelt erhalten würden, denn dies verhindert die Besitzstandswahrung, sondern entweder im Hinblick auf eine niedrigere Eingruppierung oder aufgrund der Tatsache, dass bei Anwendung der Tariflichen Entsprechung die neuen Tarifentgelte insbesondere bei den höheren Angestellten unter den vorherigen Tarifgehältern lagen. Dementsprechend werden in allen Untersuchungsbetrieben die vormaligen $\mathrm{Ge}$ haltsgruppen T4, T5, T6 (bei den technischen Angestellten) bzw. K4, K5, K6 (bei den kaufmännischen Angestellten) zu den „Verlierern“ gezählt. Besonders getroffen wurde die Belegschaft in Unternehmen, in denen der Akademikeranteil sehr hoch liegt und die Forschungs- und Entwicklungsabteilungen gegenüber den Produktionsbereichen dominieren. Die individuelle Betroffenheit der Beschäftigten geht somit weniger auf eine tatsächliche Einkommenseinbuße zum Zeitpunkt der ERAEinführung zurück als vielmehr auf zwei davon entkoppelte Veränderungen: Zum einen mussten die Beschäftigten bei der folgenden Tariferhöhung feststellen, dass die Ausgleichszulage zur Hälfte auf das Volumen der Tariferhöhung angerechnet wird, sie somit nur zur Hälfte von der Entgelterhöhung profitieren. Zum zweiten sahen sich insbesondere Personen aus dem Kreis der technischen Angestellten, die sich gerade in technologiegeprägten Unternehmen zu den Leistungsträgern des Betriebs rechnen, in ihrem beruflichen Selbstverständnis bzw. in der Wertschätzung ihrer Qualifikation infrage gestellt. In Abhängigkeit vom Leistungsprogramm des Unternehmens ist in Unternehmen, deren Kompetenz hauptsächlich in der Forschung und Entwicklung und weniger in der Produktion angesiedelt ist, ein großer Teil der Belegschaft von dieser Anpassung der Grundentgelte betroffen.

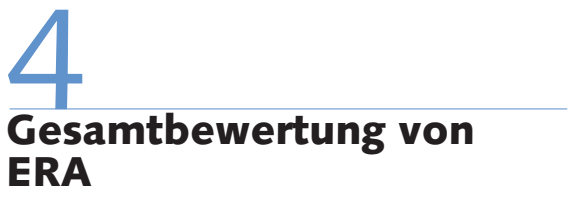

\subsection{VOR- UND NACHTEILE AUS SICHT DER BEFRAGTEN}

Insgesamt ist die "Schnittmenge“ der Bewertungen von ERA seitens der beiden verantwortlichen Betriebspartner recht groß. Von den Personalleitungen und Betriebsräten, aber auch von den Beschäftigten wird eine übereinstimmende positive Gesamtbewertung des ERA vorgenommen: eine gerechtere Entgeltpolitik und Transparenz für die Beschäftigten aufgrund der systematischen Tätigkeitsbeschreibungen; eine höhere Gerechtigkeit und Durchlässigkeit durch einheitliche Funktionsbeschreibungen; ein geringerer Aufwand bei der Eingruppierung durch die Aufhebung der Trennung zwischen Lohn- und Gehaltsempfängern; eine bessere Eingruppierung von Mitarbeitern im gewerblichen Segment entsprechend der Ausbildung und Berufserfahrung; die Förderung der Weiterbildungsbereitschaft und bessere Karriereperspektiven; bessere Differenzierungsmöglichkeiten in den höheren Entgeltgruppen durch zusätzliche Entgeltgruppen bzw. Zusatzstufen. Mit ERA konnte nach dem Urteil beider Betriebsparteien das Ziel erreicht werden, eine Bereinigung der historisch entstandenen Entgeltstrukturen in den Betrieben vorzunehmen und „Altlasten" zu beseitigen.

Auch der Großteil der Beschäftigten sieht in ERA einen sinnvollen Tarifabschluss. Dies gilt häufig sogar dann, wenn die Beschäftigten persönliche Nachteile für sich sehen. Sie erkennen damit an, dass der Wert einer tariflichen Neuregelung für eine ganze Branche und Region nicht von Vorteilen des Einzelnen abhängt, und sie sehen ihre individuelle Einkommenssituation zudem durch die Besitzstandswahrung und die betriebliche Aushandlung ausreichend geschützt. Positiv wurde auch bewertet, dass die Mitarbeiterinnen und Mitarbeiter in vielen Fällen mit ERA erstmals ihre Funktionsbeschreibungen schriftlich erhalten haben. Damit sind die Grundlagen und Entwicklungsperspektiven für die Kolleginnen und Kollegen transparent geworden. Besonders im gewerblichen Segment wird nun über die reine Berufsausbildung hinaus eine bessere Berücksichtigung von
Weiterbildung und Berufserfahrung ermöglicht, sodass die Weiterbildungsbereitschaft gesteigert wird und Aufstiegsmöglichkeiten gegeben sind.

Insgesamt gelang es den Betriebsparteien, in den aktualisierten Arbeitsbewertungen widersprüchliche, uneinheitliche, unzureichende oder auch fehlende Beschreibungen zu vermeiden. Zudem bot ERA in einer Phase der Stabilisierung der Betriebsstrukturen nach zum Teil turbulenter Firmenentwicklung die Chance, einen betrieblichen Neuanfang in der Entgeltsystematik und -gerechtigkeit zu wagen. Eine Zielsetzung für die schnelle ERA-Einführung war die Hoffnung beider Betriebsparteien, mittels der „Tariflichen Entsprechung" eine reibungslose Umsetzung zu erreichen und Konflikte aus dem Unternehmen herauszuhalten. Für die Betriebsräte bestand damit zudem die Chance, das erreichte Lohnniveau in der Firma zu erhalten und das bestehende Gehaltsgefüge gegen drohende Abgruppierung zu sichern.

Von den Personalleitungen wird als einziger Nachteil hervorgehoben, dass es mit ERA nicht gelungen sei, die gesamte Tariflandschaft in der Bundesrepublik zu vereinheitlichen. Noch immer würden zu viele einzelne Tarifverträge in der BRD gelten. Insbesondere in Konzernverbünden mit Standorten in mehreren Bundesländern oder beim Arbeitsplatzwechsel von Beschäftigten über Ländergrenzen hinweg ergeben sich Veränderungen in Eingruppierung und Entgelt, die evtl. auch persönliche Nachteile mit sich bringen.

\subsection{WECHSELWIRKUNG ZWISCHEN ENTGELTSYSTEMEN UND ETABLIE- RUNG NEUER ARBEITSFORMEN}

Über die Neuthematisierung der Entgeltrelationen hinaus erhoffte sich insbesondere die Gewerkschaft von ERA positive Effekte für die Einführung moderner, für die Humanisierung der Arbeit fortschrittlicher Ansätze der Arbeitsorganisation. Insbesondere die Gratifizierung der Übernahme dispositiver Funktionen mittels der Zusatzstufen sowie die einheitliche Entgeltsystematik sollten Impulswirkungen für die Einführung neuer Arbeitsformen und Produktionskonzepte geben. Man erhoffte sich dadurch eine Auflösung bisheriger Trennlinien zwischen Arbeitsvorbereitung, Produktionsplanung und -steuerung einerseits und der Produktion andererseits. Mit ERA 
war zugleich von Gewerkschaftsseite die Zielsetzung verbunden, die bislang von den Beschäftigten zwar abgeforderten, aber nicht entgoltenen Kompetenzen zur Sicherstellung einer reibungslosen Produktion zu bezahlen - und dadurch den Unternehmen einen Anreiz zu geben, derartige Fähigkeiten und Fertigkeiten auch durch geeignete Arbeitsformen zur Wirkung kommen zu lassen.

Diese Hoffnungen der IG Metall haben sich allerdings nicht erfüllt. Moderne Produktionskonzepte und Arbeitsformen haben in den Betrieben bereits vor der ERAEinführung bestanden und existieren auch weiterhin. Die Umsetzung des ERA war in keinem der untersuchten Fälle ursächlich für organisatorische Umstellungen. Dasselbe gilt für personelle und personalpolitische Folgen; in keinem der Untersuchungsbetriebe ergaben sich durch ERA quantitative bzw. qualitative Beschäftigungseffekte oder personalpolitische Auswirkungen. ERA erfüllte also nicht die gewerkschaftlichen Hoffnungen auf eine Impuls- und Inkubatorfunktion für moderne Arbeitsformen und neue Produktionstypen. Positiv gewendet ist allerdings festzuhalten, dass ERA in keinem Fall als Barriere oder blockierend gegen eine Modernisierung von Arbeits- und Produktionsmodellen gewirkt hat.

\subsection{GEWERKSCHAFTS- UND BETRIEBSRATSRÜCKHALT: SOLIDARISIERUNG VERSUS DISTANZIERUNG}

Mit den sich aus der ERA-Anwendung für unterschiedliche Belegschaftsgruppierungen ergebenden persönlichen, zum Teil einkommensrelevanten Vor- oder Nachteilen ist die Frage verknüpft, wie sich solche Erfahrungen auf die Einschätzungen zum Betriebsrat und zur Gewerkschaft auswirken.

(1) Rückhalt des Betriebsrats: Wie für ein Vorhaben wie ERA zu erwarten, kam es anfangs zu Irritationen in den Belegschaften, welche Konsequenzen sich für die Einkommenssituation ergeben könnten. Dabei werden die Betriebsräte, die eine erfolgreiche ERA-Einführung als ihre originäre Aufgabe verstanden, mit den ERA-Maßgaben und -Auswirkungen identifiziert. Zieht man als Indiz die Selbsteinschätzung der befragten Betriebsräte und die Fremdeinschätzung des Betriebsratshandelns seitens der Mitarbeiter heran, hat sich durch ERA in allen Untersuchungsbetrieben die Bedeutung und Wahrnehmung des Betriebsrats in der Belegschaft deutlich verbessert. Die Rolle der Betriebsräte während der Einführung von ERA wird von den Mitarbeiterinnen und Mitarbeitern explizit gewürdigt: Er habe eine positive Rolle gespielt, indem er sich für die Mitarbeiter eingesetzt und Vorteile für die Beschäftigten ausgehandelt habe. Diese Einschätzung wird in den meisten Fällen auch von Mitarbeitern geteilt, die sich durch die ERAUmsetzung als benachteiligt einschätzen oder über die als Streitfall in der Paritätischen Kommission verhandelt wurde. Hier zeigt sich, dass schon die Thematisierung des eigenen Falls dem Arbeitgeber gegenüber, durch den Betriebsrat vertreten, gewürdigt wird, da sich die Mitarbeiter in ihren individuellen Interessen ernst genommen fühlen.

(2) Gewerkschaftlicher Organisationsgrad. Die guten Erfahrungen mit der Arbeit des Betriebsrats führten allerdings nicht zu Gewerkschaftsbeitritten bzw. einer Erhöhung des betrieblichen Organisationsgrads. Dieser ist in zwei der drei Untersuchungsbetriebe, in denen der Angestelltenanteil überwog, in den letzten Jahren konstant geblieben und hat sich auch mit der ERA-Einführung nicht geändert. In dem Untersuchungsbetrieb mit eigener Produktion aus der Automobilindustrie sank er hingegen zeitweise von ehedem $45 \%$ auf ca. $32 \%$, was von den Gesprächspartnern auf die anfänglichen Befürchtungen der Beschäftigten zurückgeführt wird, es drohe eine $\mathrm{Ab}$ senkung der übertariflichen Bezahlung auf das Tarifniveau - manche Überschreiter seien daher sofort aus der Gewerkschaft ausgetreten. Doch auch in diesem Fall hat sich der Organisationsgrad aufgrund der betrieblichen Informationspolitik und der Besitzstandswahrung nunmehr wieder stabilisiert.

(3) Im Hinblick auf den Rückhalt der Gewerkschaft bei den Betriebsräten lassen sich mit der ERA-Umsetzung positive Wirkungen feststellen. Gerade im teilweise von Unsicherheit geprägten Umsetzungsprozess eines Tarifwerks kommt der Gewerkschaft bzw. den örtlichen Bevollmächtigten eine phasenweise deutlich gestärkte Bedeutung für die Erläuterung von Tarifdetails, die Bereitstellung von Umsetzungshilfen und die Aufklärung über entscheidende Meilen- steine zu. Die schon immer sehr guten Kontakte zwischen Gewerkschaft und Betriebsrat wurden in den Untersuchungsfällen während der ERA-Einführung deutlich intensiviert, haben sich aber nach $\mathrm{Ab}$ schluss der ERA-Einführung wieder auf ein „normales Maß“ eingependelt; sie sind nun nicht besser oder schlechter als vor der Einführung.

(4) Ein zusätzlicher vorteilhafter ERA-Effekt betraf eine stärkere Vernetzung von Betriebsräten untereinander. In einem der Untersuchungsbetriebe wurden bestehende Kontakte zu den anderen Betriebsräten in der Region deutlich ausgeweitet und institutionalisiert. Es finden nunmehr feste Betriebsratsstammtische statt, um eine wechselseitige Information und Hilfestellung auch in über ERA hinausgehenden Fragen zu intensivieren.

\section{5 \\ Resümee: Verbands- politische Perspektiven durch ERA}

Beide Tarifparteien verbanden mit den ERA-Abschlüssen weitreichende Hoffnungen auf eine Bereinigung der historisch gewachsenen betrieblichen Entgeltstrukturen. Zugleich jedoch wurde befürchtet, dass mit der Thematisierung von Arbeitsbewertungen und Entgeltgrundlagen und aufgrund der neuartigen Tarifbestimmungen umfangreiche Konflikte in die Betriebe der Metall- und Elektroindustrie getragen werden könnten (Huber/Schild 2004). Mittlerweile liegen von den Gewerkschaften und Arbeitgeberverbänden, aber auch von Wissenschaftlern Zwischenberichte und Praxisbeispiele vor, die darauf hindeuten, dass sich die besonders kritischen Einführungsphasen und die Betroffenheit von Belegschaftsgruppen in den unterschiedlichen Tarifgebieten durchaus gleichen. Die Unternehmen, die ERA bereits eingeführt haben, erkennen nach anfänglichen Berührungsängsten zum weit überwiegenden Teil Vorteile für das Unternehmen und die Beschäftigten sowie ein gewachsenes Vertrauen in tarifliche Regelungen (vgl. die Praxisbeispiele aus unterschiedlichen Tarifgebieten: Hofmann/Rösner 2007; Bossemeyer/Mackau 2006; Kuhlmann/Sperling 2008; Brossardt/Mantl 2007). Es wird aber auch von konfliktreicheren Umsetzungs- 
prozessen mit hohen Anteilen von Überschreitern und entsprechender Unzufriedenheit unter der Gewerkschaftsklientel berichtet (Bahnmüller/Schmidt 2008; dies. in diesem Heft). Hier soll vor dem Hintergrund der empirischen Befunde aus Thüringen abschließend eine sozialwissenschaftliche Bewertung der interessenpolitischen Perspektiven von ERA im Hinblick auf die häufig diagnostizierte Erosion des Tarifvertragssystems vorgenommen werden. Es geht um die Frage, ob sich in den Befunden eine Gegenbewegung zur Erosion erkennen lässt bzw. inwieweit ERA eine weitergehende Verbetrieblichung von Aushandlungsgegenständen mit sich bringt.

In den letzten Jahrzehnten ließ sich in Gesamtdeutschland bekanntlich ein Trend zur Abnahme kollektiver Verbindlichkeit und verbandlicher Mobilisierungsfähigkeit feststellen, der als Wandel der für Deutschland charakteristischen Tariflandschaft bezeichnet wurde und sich im Grunde durch zwei Elemente auszeichnete: Erstens nahm die quantitative Bedeutung und Reichweite von flächendeckenden Verbandstarifverträgen aufgrund abnehmender Tarifbindung ab (Ellguth 2004; Hinke et al. 2002). Zweitens wandelte sich der qualitative Charakter der Tarifverträge durch Flexibilisierung, Differenzierung und Öffnungsklauseln (Bispinck 2003; Kohaut/Schnabel 2003). In der sozialwissenschaftlichen und gewerkschaftsnahen Forschung werden derartige Trends auch unter dem Etikett der "Verbetrieblichungsthese" debattiert: Zunehmend würden tarifvertragliche Regelungsnotwendigkeiten von der tariflichsektoralen Ebene auf die Ebene von Unternehmen bzw. Betrieben verlagert (vgl. Deiß 2000).

Im ERA-Kontext ist hier eine widersprüchliche Tendenz festzustellen:

(1) Einerseits lässt sich ein Trend zur Verbetrieblichung von Aushandlungsverfahren durch Übertragung von Einigungsverfahren auf die Betriebsebene in den neuen ERA-Tarifverträgen feststellen. Es kommt zum Ersten zu einer Verbetrieblichung der Entscheidung über Entgeltgrundsätze, da die Tarifverträge eine breite Palette von Entgeltmodellen zulassen. In diesem Sinn haben die Tarifvertragsparteien den Betrieben die Entscheidung überantwortet, weiterhin die bisherigen betrieblichen Vereinbarungen zum Leistungsentgelt anzuwenden oder mit dem Zeitpunkt der ERA-Einführung die jeweiligen Regelungen des
Entgeltrahmenabkommens zu übernehmen. Auch bezüglich der Öffnung für die Festlegung betrieblicher Entgeltregelungen in Betriebsvereinbarungen, wie sie ERA vorsieht, kann von einer Verbetrieblichung gesprochen werden - allerdings in Form einer ,kontrollierten Dezentralisierung“" (Bispinck 2004), da abweichende betriebliche Regelungen der Zustimmung der Tarifvertragsparteien bedürfen. Zum Zweiten werden formale, substanzielle Definitionen ersetzt durch eine Prozeduralisierung der Regulierung, ablesbar z. B. an der im Tarifvertrag vorgesehenen Einrichtung von $\mathrm{Pa}-$ ritätischen Kommissionen in den Betrieben. Mit Hilfe dieser Paritätischen Kommissionen sollen Konflikte bzw. Auslegungsunterschiede zwischen Arbeitgebern und Arbeitnehmern unmittelbar vor Ort gelöst werden anstatt in Schlichtungsverfahren der Tarifparteien.

Gerade bei diesem Thema sprechen die empirischen Befunde aus Thüringen allerdings gegen eine umstandslose Gleichsetzung von Verbetrieblichung und Erosion des Tarifvertragssystems. Denn gerade die Paritätischen Kommissionen haben sich in den Betrieben als durchgängiges Erfolgsmodell herausgestellt. Sie haben einerseits einen Einigungsdruck zwischen Unternehmensleitung und Betriebsrat angestoßen und andererseits zu einem „Kleinarbeiten“ von Einsprüchen der Beschäftigten im Betrieb geführt. Dies hat nicht nur die Akzeptanz von ERA, sondern auch den Rückhalt der Betriebsräte in den Belegschaften gefördert.

(2) Andererseits lassen die bisherigen Erfahrungsberichte aus Unternehmen den Schluss zu, dass mit der gemeinsamen, zeitlich koordinierten Initiative zur ERA-Einführung nicht nur in Thüringen ein vorheriger Wildwuchs an betrieblichen Eingruppierungs- und Leistungsentgeltmodellen bereinigt und die tarifpolitische Ordnungsfunktion (wieder) gestärkt wurde. Aus der Interessenlage der Gewerkschaft kommt der Vorteil hinzu, dass es mit ERA erstmals gelungen ist, durch die tarifvertragliche Definition der Entgelte als Leistungsentgelte die Entgeltregelungen maßgeblicher Belegschaftsteile - der vormals Angestellten - in die Mitbestimmung durch die Betriebsräte einzubeziehen. Sollten diese Tendenzen über die betrachteten Einzelfälle hinausgehen, würde sich darin in der Tat eine Rückkehr zu vereinheitlichten Tarifregularien im Entgeltfeld und eine
Abkehr von den Abweichungsbestrebungen ausdrücken.

Für das Tarifgebiet Thüringen als ostdeutsches Tarifgebiet liegt eine besondere Situation insofern vor, als die geringe Tarifbindung hier - im Gegensatz zu westdeutschen Regionen - ausschließlich darauf zurückzuführen ist, dass neu gegründete Unternehmen in der Regel nicht dem Arbeitgeberverband beitreten. Es handelt sich somit eher um eine Tarifabstinenz und weniger um eine Tarifflucht. Vor diesem Hintergrund verfolgten beide Tarifparteien in Thüringen auch die Zielsetzungen einer Stärkung des Flächentarifvertrags und einer tarifvertragskonformen ERA-Umsetzung in den Betrieben. Mit ERA war zudem seitens des Arbeitgeberverbands die Hoffnung verbunden, Unternehmen (wieder oder neu) in die Tarifbindung zu bringen. Zwei sehr vorsichtig zu wertende Indizien scheinen die Aussage zu stützen, dass der im Osten Deutschlands insbesondere in der ersten Hälfte der 1990er Jahre zum Teil dramatisch verlaufene Trend zu einer sehr geringen Tarifbindung von Unternehmen und zur Nicht-Anwendung von Tarifregelungen in Thüringen zumindest gebremst, wenn nicht gar gestoppt werden konnte. In diese Richtung weist zum Ersten die Tatsache, dass in Thüringen der Organisationsgrad von Unternehmen im Verband in den letzten Jahren konstant ist, während der OT-Verband (Betriebe ohne Tarifbindung) stagniert. Zum Zweiten scheint es in Thüringen gelungen zu sein, die tarifgebundenen Unternehmen zur vollständigen Übernahme des ERA zu veranlassen. Bis Ende 2008 haben $90 \%$ der tarifgebundenen Unternehmen ERA umgesetzt. Die restlichen $10 \%$ haben in Abstimmung mit den Tarifvertragsparteien tarifvertragsgemäß eine spätere Einführung nach 2008 vereinbart. Auch $20 \%$ der OT-Firmen haben das Entgeltrahmenabkommen übernommen.

Die Befunde der empirischen Begleitforschung in Thüringen lassen darauf schließen, dass mit der ERA-Einführung die kollektiven Interessenvertretungsverbände gestärkt aus dem Umsetzungsprozess hervorgingen. Die Gewerkschaften konnten den Organisationsgrad in den Betrieben stabilisieren, wenn auch nicht ausbauen. Die gestiegene Zustimmung der Belegschaften zur Betriebsratsarbeit zeigt eine Stärkung der Institution Betriebsrat an.

(3) Die Tarifvertragsparteien haben große betriebliche Spielräume eröffnet und durch 
Wahl- und Entscheidungsoptionen einen großen Teil der Verantwortung für die Entgeltgestaltung den Betriebsparteien übertragen. Während dies einerseits Forderungen nach einer betriebsnahen Tarifpolitik Rechnung trägt, hängt eine erfolgreiche Interessenpolitik im Sinne der Beschäftigten damit andererseits sehr viel stärker von einer mit der Gewerkschaft abgestimmten Position und ausreichenden Machtressourcen der Betriebsräte ab. Gewerkschaftspolitisch erfordert eine betriebsnähere Tarifpolitik folglich eine weitergehende Verknüpfung von Tarif- und Betriebspolitik, bei der neben der Mitgliederentwicklung zur Erhöhung des gewerkschaftlichen Or- ganisationsgrades in den Betrieben die ,gezielte Verbesserung der betrieblichen Umsetzungsmöglichkeiten und -instrumente für Tarifverträge" geleistet wird (Huber et al. 2005, S. 659; Urban 2005; Iwer/Wagner 2005). Mitentscheidend wird in diesem $\mathrm{Zu}$ sammenhang sein, inwieweit sich die ERAUmsetzung letztlich als Instrument der Mitgliedergewinnung und -entwicklung (Huber et al. 2005) für die Gewerkschaften und Arbeitgeberverbände in der Metallund Elektroindustrie nutzen lässt. Dies hängt sicherlich auch von der Akzeptanz der ERA-Einführung und der Beurteilung der Rolle von betrieblichen und überbetrieblichen Interessenvertretungsorganisa- tionen durch die Belegschaft und die Betriebsleitungen ab. Die Erfahrungen in den Untersuchungsbetrieben verweisen darauf, dass zwar eine derartige Mitgliederrekrutierung durchaus gelingen könnte, hierfür aber die Betriebsräte und Gewerkschaftsfunktionäre diese Aufgabe ausdrücklich in ihren Zielekanon einbeziehen müssten. Demgegenüber stand bisher die praktische ERA-Umsetzung so weit im Vordergrund, dass die Gefahr besteht, Chancen zur Stärkung der eigenen Machtbasis in den Unternehmen sowie der Verhandlungsfähigkeit von Betriebsräten zu vergeben.

\section{LITERATUR}

Bahnmüller, R./Schmidt, W. (2008): Der ERA und seine Umsetzung. Erfahrungen aus Baden-Württemberg, in: Bispinck, R. (Hrsg.): Verteilungskämpfe und Modernisierung, Wiesbaden, S. 78-108 Bispinck, R. (2003): Das deutsche Tarifsystem in Zeiten der Krise - Streit um Flächentarif, Differenzierung und Mindeststandards, in: WSI-Mitteilungen 7, S. 395-404

Bispinck, R. (2004): Kontrollierte Dezentralisierung der Tarifpolitik Eine schwierige Balance, in: WSI-Mitteilungen 5, S. 237-245

Bossemeyer, A./Mackau, D. (2006): Die betriebliche ERA-Einführung im Nordverbund, in: Angewandte Arbeitswissenschaft 188, S. 20-38 Brossardt, B./Mantl, G. (2007): Aktueller Stand der ERA-Einführung in Bayern, in: Angewandte Arbeitswissenschaft 194, S. 1-11

Deiß, M. (2000): Betriebsrat - Quo vadis? Interessenvertretung in vernetzten Wertschöpfungsketten, in: Klitzke, U./Betz, H./Möreke, M. (Hrsg.): Vom Klassenkampf zum Co-Management, Hamburg, S. 117-146 Ellguth, P. (2004): Erosion auf allen Ebenen? Zur Entwicklung der quantitativen Basis des dualen Systems der Interessenvertretung, in: Artus, I./ Trinczek, R. (Hrsg.): Über Arbeit, Interessen und andere Dinge, München/Mering, S. 159-179

Haipeter, T./Schilling, G. (2006): Arbeitgeberverbände in der Metall- und Elektroindustrie. Tarifbindung, Organisationsentwicklung und Strategiebildung, Hamburg

Hinke, R./Röbenack, S./Schmidt, R. (2002): Diesseits und jenseits des Tarifvertrages. Die Gestaltung der Lohn- und Leistungsbedingungen in der ostdeutschen Metall- und Elektroindustrie, Studie der Otto Brenner Stiftung, Berlin

Hofmann, A./Rösner, U. (2007): Die ERA-Umsetzung in NordrheinWestfalen: Eine Halbzeitbilanz, in: Angewandte Arbeitswissenschaft 193, S. 1-18
Huber, B./Burkhard, O./Klebe, T. (2005): Tarifpolitik ist Betriebspolitik, Betriebspolitik ist Tarifpolitik, in: WSI-Mitteilungen 11, S. 656-662 Huber, B./Schild, A. (2004): Die neuen Entgeltrahmentarifverträge, in: WSI-Mitteilungen 2, S. 102-105 Iwer, F./Wagner, H. (2005): Grenzen der Vermarktlichung. Elemente einer aktivierenden Arbeits-, Betriebs- und Tarifpolitik, in: Detje, R./ Pickshaus, K./Urban, H.-J. (Hrsg.): Arbeitspolitik kontrovers, Hamburg, S. 112-124 Institut der Wirtschaft Thüringens (IWT) (2007): Die Zukunft des Arbeitsmarktes in Thüringen. Jahresumfrage der Verbände VWT, AGVT, VMET, Erfurt

Kohaut, S./Schnabel, C. (2003): Zur Erosion des Flächentarifvertrags, in: Industrielle Beziehungen 2, S. 193-219

Kuhlmann, M./Sperling, H. J. (2008): Neue (A)ERA? Zur Umsetzung des neuen Entgelt-Rahmentarifvertrags in der Metall- und Elektroindustrie Niedersachsens, in: Mitteilungen aus dem SOFI 3, April, S. 5-7

Meine, H./Sadowsky, R./Schulz H. (2006): "Wird bezahlt, was verlangt wird?" Eingruppierung, Arbeitsbewertung, Qualifikation, in: Ehlscheid, C./Meine, H./Ohl, K. (Hrsg.): Handbuch Arbeit - Entgelt - Leistung, Frankfurt/Main, S. 98-201

Schmierl, K. (2008): Eine neue AERA in Thüringen - Einführung und Umsetzung des Entgeltrahmenabkommens in ausgewählten Pilotbetrieben, ISF Forschungsberichte, München

Urban, H.-J. (2005): Wege aus der Defensive. Schlüsselprobleme und -strategien gewerkschaftlicher Revitalisierung, in: Detje, R./Pickshaus, K./ Urban, H.-J. (Hrsg.): Arbeitspolitik kontrovers, Hamburg, S. 187-212 